\title{
Middle cerebral artery anatomical variations and aneurysms: a retrospective study based on computed tomography angiography findings
}

\author{
P. Brzegowy ${ }^{1}$, J. Polak², J. Wnuk², B. Łasocha ${ }^{1}$, J. Walocha ${ }^{3}$, T.J. Popiela ${ }^{1}$ \\ ${ }^{1}$ Department of Radiology, Jagiellonian University Medical College, Krakow, Poland \\ 2Students' Scientific Group at the Department of Radiology, Jagiellonian University Medical College, Krakow, Poland \\ ${ }^{3}$ Department of Anatomy, Jagiellonian University Medical College, Krakow, Poland
}

[Received: 5 July 2017; Accepted: 26 October 2017]

Background: Anatomical variations of the middle cerebral artery (MCA) are an important clinical issue, due to high prevalence of intracranial aneurysms. Anatomical variations of vessels can lead to higher shear stress, which is thought to be the main factor leading to aneurysm formation and consequently to higher prevalence of aneurysms. The aim of this study was to evaluate anatomy of the MCA; to classify MCA aneurysms using computed tomography angiography and to correlate anatomical variations of MCA and circle of Willis with prevalence of MCA aneurysms.

Materials and methods: Two hundred and fifty patients without MCA aneurysms and 100 patients with unruptured MCA aneurysms were qualified for the study, with exclusion of patients after MCA clipping. Four aspects of MCA anatomy were evaluated: division point, its relation to the genu, distance to $M 1$ division and the genu and domination of post-division trunks.

Results: Middle cerebral artery bifurcation was found in $86.2 \%$ and trifurcation in $13.8 \%$ of the cases. $78.4 \%$ of MCAs divided before the genu, $19.2 \%$ in the genu and $2.4 \%$ after the genu. Upper branch domination was seen in $26 \%$, lower branch in $25.4 \%$, middle branch in $4 \%$ and no domination in $44.6 \%$ of the cases. In the study group 116 aneurysms were found. 86.2\% of the aneurysms were located in M1 division point, 6.9\% in M2 segment, 3.4\% near lenticulostriatae arteries and 3.4\% near early cortical branches. The only anatomical variation, which had significantly higher prevalence in patients with left MCA aneurysms, was domination of upper post-division trunk of MCA. No other statistically significant differences in circle of Willis and MCA variations were found between patients with aneurysms and without them.

Conclusions: The most common configuration of MCA is bifurcation before the genu with no dominating post-division trunk. Incidence of MCA aneurysms is not correlated with anatomical variations of MCA and the circle of Willis. (Folia Morphol 2018; 77, 3: 434-440)

Key words: middle cerebral artery, circle of Willis, intracranial aneurysms 


\section{INTRODUCTION}

Morphology and anatomical variations of the middle cerebral artery (MCA) are an important clinical issue, mainly due to the high prevalence of intracranial aneurysms. MCA is the second most common location for intracranial aneurysms, after anterior communicating artery. The share of MCA aneurysms in all intracranial aneurysms ranges from $20 \%$ to $43 \%[5,9,17]$. Thorough knowledge of anatomical variations of the proximal segments of the MCA makes it easier to plan and provide treatment, both surgical and endovascular. With introduction of interventional stroke treatment, knowledge of MCA anatomy becomes even more important, as it is the most common location of clot available to treatment by mechanical thrombectomy [16].

Middle cerebral artery is phylogenetically the youngest intracranial artery. Its evolution is closely related to the development of cerebrum (frontal, temporal and parietal lobes). Cortical vessels, which will form MCA, appear on lateral aspects of hemispheres before infolding and deepening of hemispheres occurs, leading to formation of insula. In this period (8-12 weeks' gestation) these cortical vessels originate directly from terminal segment of internal carotid artery (ICA). When insula develops, the arteries penetrate the forming Sylvian fissure. Also, in this period they form MCA, which continues to develop up to birth [9].

Pathogenesis of aneurysms is not completely understood. Shear stress, putting pressure on arterial walls, is thought to be the main factor leading to their formation. It causes mechanical damage to the wall and eventually leads to vascular malformation. Atheromatous plaques are the main factor leading to increased shear stress. Some developmental variations can also increase shear stress. Other factors influencing aneurysm formation include: older age, female sex, hypertension, hereditary connective tissue disorders, alcohol consumption and smoking $[8,18]$.

Developmental disorders of intracranial vessels can also be related to higher prevalence of aneurysms. Anatomical variations of the circle of Willis are caused by its arrested development. Underdeveloped arteries are weakened and at higher risk of aneurysms formation [13]. The anatomical variations can also lead to increased shear stress in connected arteries. For example, association of hypoplasia and aplasia of A1 segment of anterior cerebral artery and anterior communicating artery aneurysms is well proven [11].

The aim of the study was to evaluate anatomy of MCA, prevalence of MCA aneurysms and to classify them using computed tomography angiography (CTA). It was also attempted to correlate anatomical variations of MCA and the circle of Willis with prevalence of MCA aneurysms.

\section{MATERIALS AND METHODS}

To evaluate anatomy of MCA, 266 consecutive CTA findings in the patients without MCA aneurysms performed from March 2015 to February 2016 in the University Hospital in Krakow were retrospectively analysed. Two hundred and fifty patients (134 women and 116 men) were included in the study. Mean age of the patients was 52.1 years, with a standard deviation of \pm 10.2 . The age range was $24-83$ years. Sixteen patients were excluded from the study: 5 with a stroke in MCA territory, 8 patients with occluded MCA, 2 patients with unknown identity, and 1 patient due to unsatisfactory quality of the CTA images.

To evaluate MCA aneurysms, all reports of CTA head scans performed from September 2013 to February 2016 were read. One hundred and forty-five patients with MCA aneurysm were identified. Forty-five patients were excluded: 37 with clipped aneurysm, 1 with coiled aneurysm, 4 with stroke in MCA territory, 2 with giant aneurysms and 1 with intracerebral haematoma, as these would hinder accurate MCA anatomy assessment. CTA images of 100 patients were analysed and revealed 116 MCA aneurysms. Mean age of the patients was 58.7 with standard deviation of \pm 17.6 years. The age range was $16-93$ years. The study group consisted of 70 women and 30 men.

Computed tomography (CT) was performed using multi-row CT scanner (GE Optima CT 660). The parameters of CT study were: exposure factors $120 \mathrm{kV}, 320 \mathrm{~mA}, 120 \mathrm{mAs}$; slice thickness $-0.625 \mathrm{~mm}$.

The data were sent to the workstation equipped with software for obtaining three-dimensional volume rendering (3D VR) and maximum-intensity projection (MIP) reconstructions in three perpendicular planes coronal, sagittal and axial.

Evaluation of MCA anatomy was performed in four steps:

1. M1 division point was determined as a point where main insular trunks converge on sagittal plane, using MIP reconstruction. Then the division point was verified on coronal and axial planes, as well as on $\mathrm{VR}$ reconstruction.

2. Next, the relation of M1 division point to genu was evaluated. Genu is a gentle upward curve of MCA trunks near the limen insulae. 


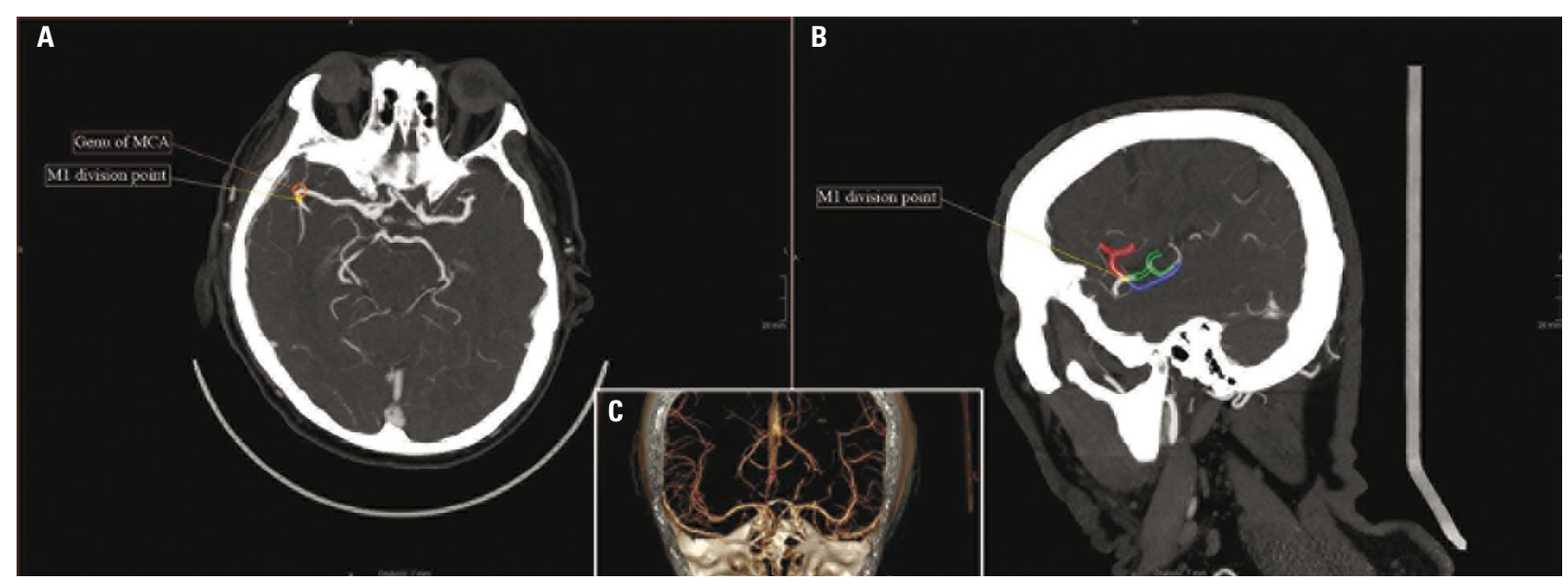

Figure 1. Radiological documentation of middle cerebral artery (MCA) anatomy evaluation; A. Maximum intensity projection (MIP) reconstruction of sagittal plane, showing MCA division point; B. MIP reconstruction of transverse plane, showing MCA genu and measurement of M1 length. MCA domination was assessed in both planes; C. Three-dimensional reconstruction useful in more complicated cases.

3. The distance from ICA to M1 division was measured between facing angles of both divisions on axial plane.

4. Domination of post-division trunks was evaluated on sagittal and axial planes. No domination was determined, when there was no significant difference in diameter of MCA trunks after M1 division. In evaluation of trunk domination, the area of vascularisation was also factored in (for lower trunk: temporal, temporooccipital and angular areas; for upper trunk: orbitofrontal and posterior parietal areas) [2].

Radiological documentation of MCA anatomy evaluation is presented in Figure 1.

Aneurysms were assigned to four groups, according to Elsharkawy et al. [2] classification: (1) M1 lenticulostriate arteries aneurysms, (2) M1 early cortical branches aneurysms, (3) M1 bifurcation aneurysms, (4) distal M2 aneurysms.

\section{RESULTS}

\section{MCA anatomy}

Middle cerebral artery bifurcation was identified in $86.2 \%$ (95\% confidence interval $[\mathrm{Cl}] 83.2-89.2)$ of the cases, trifurcation in $13.8 \%(95 \% \mathrm{Cl} 10.8-16.8)$, and tetrafurcation in 1 patient [15]. 78.4\% (95\% Cl 74.8-82) of the cases divided before the genu. M1 division in the genu was found in $19.2 \%$ (95\% Cl 15.7-22.6) of the cases and after the genu in $2.4 \%$ (95\% Cl 1.1-3.7). No domination of post-division trunks was seen in $44.6 \%$ (95\% Cl 40.2-49) of the cases. Upper branch dominated in $26 \%$ (95\% Cl 22.1-29.9), lower branch in $25.4 \%$ (95\% Cl 21.6-29.2), and middle branch in $4 \%(95 \% \mathrm{Cl} 2.3-5.7)$ of the cases.

The distance from ICA division to M1 division measured $15.8 \mathrm{~mm}$ (95\% Cl 15.3-16.3). The distance from ICA division to MCA genu measured $24.2 \mathrm{~mm}$ (95\% $\mathrm{Cl}$ 23.9-24.5). Short M1 segment, defined as shorter than $10 \mathrm{~mm}$, was found in $16 \%$ of the cases.

Early cortical branches - arteries, which originate from M1 segment after lenticulostriate arteries and before M1 division (anterior temporal artery, ophtalmotemporal artery, temporopolar artery, uncal artery) were found in $38 \%$ (95\% Cl 33.7-42.3\%) of the cases.

Rare developmental variations of MCA were also observed: (1) accessory MCA was seen in 2 patients, in both on the left side; (2) MCA duplication was found in 5 cases ( 2 on the left side, 3 on the right side); (3) MCA fenestration was seen once, on the right side. Prevalence of anatomical variations is shown in Table 1. No statistically significant differences in MCA anatomy (both length, division point and number of branches) were found between both hemispheres and between sexes.

\section{MCA aneurysms}

In the study group 116 aneurysms were found: 60 aneurysms of right MCA, 56 aneurysms of left MCA. Figure 2 shows aneurysm distribution according to Elsharkawy et al. classification [2]. Men had 20 aneurysms on the right side and 15 on the left side; women had 40 aneurysms on the right side and 41 on the left side. Aneurysms prevalence was significantly higher in women and older people ( $p<0.05) .10$ patients $(10 \%$ 
Table 1. The most common middle cerebral artery (MCA) anatomical variations
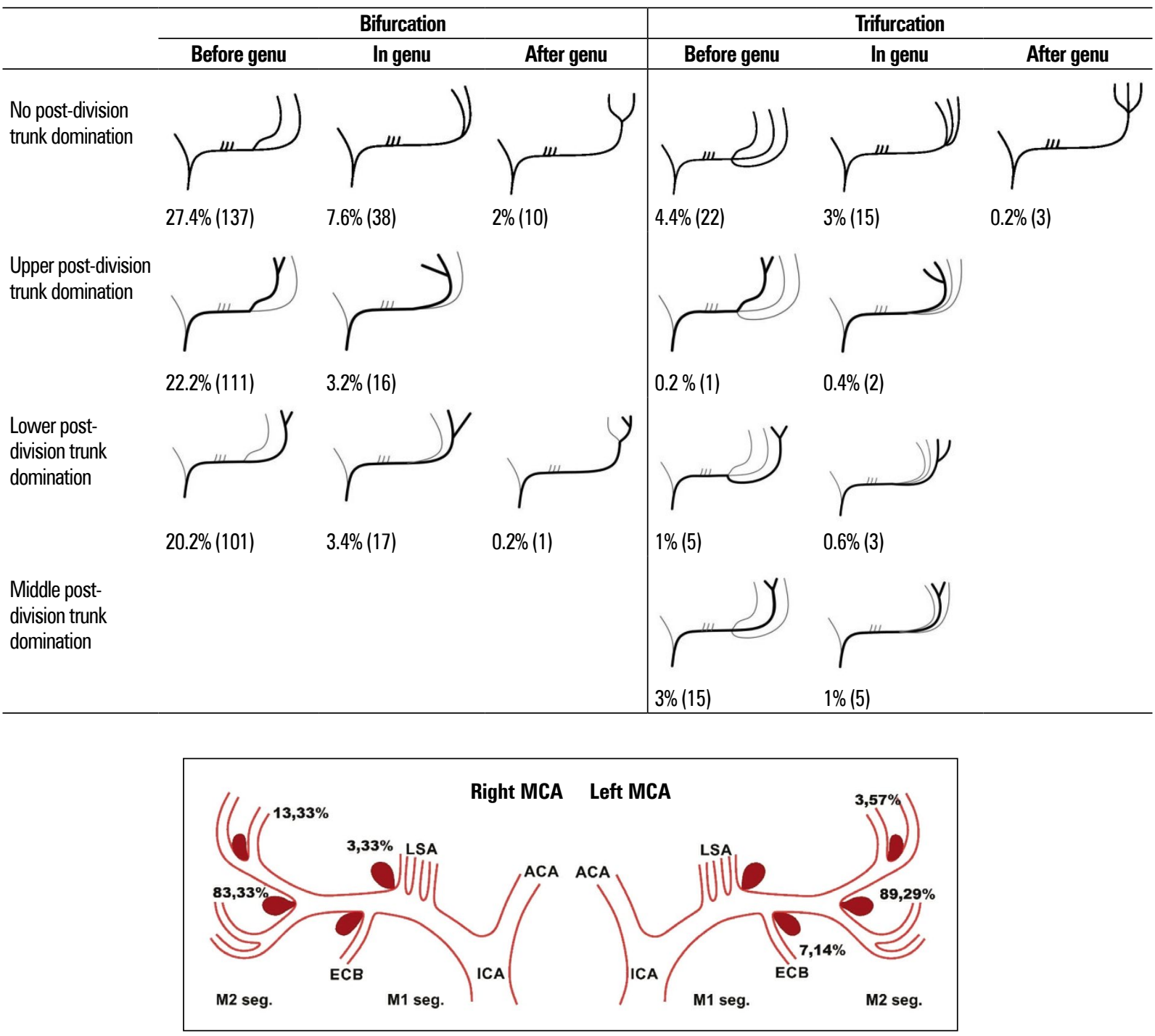

Figure 2. Prevalence of right and left middle cerebral artery (MCA) aneurysms; ACA — anterior cerebral artery; ECB — early cortical branches; ICA - internal carotid artery; LSA — lenticulostriatae arteries.

of patients; 8 women, 2 men) had mirror aneurysms in MCA division point. No mirror aneurysms in different locations were found.

Overall, most (86.2\%) of the aneurysms were located in $\mathrm{M} 1$ division point, $6.9 \%$ of the aneurysms were located in M2 segment. M1 lenticulostriate arteries aneurysms and M1 early cortical branches aneurysms each constituted $3.4 \%$ of all aneurysms found.

One of the aims of the study was to examine correlation between anatomical variations of the circle of Willis and prevalence of MCA aneurysms. The results are presented in Table 2. No statistically significant differences in the circle of Willis variations were found between patients with aneurysms and without them. Anatomical variations of the circle of Willis are shown in Figure 3.
The correlation with anatomical variations of MCA was also studied. The results are shown in Table 3. The only variation, which had significantly higher prevalence in the patients with left MCA aneurysms, was domination of upper post-division trunk of MCA. In other cases, there was no correlation of aneurysm prevalence and anatomical variations of MCA.

\section{DISCUSSION}

Studies of MCA morphology are still conducted, due to relatively numerous possible anatomical variations. Significance of CTA scans increased recently because of the progress of endovascular treatment of vascular malformations, as well as new methods of endovascular ischaemic stroke treatment. In most 
Table 2. Prevalence of circle of Willis variations in patients with and without aneurysms

\begin{tabular}{|c|c|c|c|c|}
\hline $\begin{array}{l}\text { Anatomical variant of the } \\
\text { circle of Willis }\end{array}$ & $\begin{array}{c}\text { Number of patients } \\
\text { with MCA aneurysms }\end{array}$ & $\begin{array}{c}\text { Number of patients with } \\
\text { no MCA aneurysm }\end{array}$ & $\begin{array}{l}\text { Prevalence in patients } \\
\text { with MCA aneurysms }\end{array}$ & $\begin{array}{l}\text { Prevalence in patients } \\
\text { with no MCA aneurysm }\end{array}$ \\
\hline I & 19 & 55 & $19 \%$ & $22 \%$ \\
\hline II & 3 & 14 & $3 \%$ & $5.6 \%$ \\
\hline III & 14 & 19 & $14 \%$ & $15.6 \%$ \\
\hline IV & 5 & 6 & $5 \%$ & $2.4 \%$ \\
\hline V & 27 & 46 & $27 \%$ & $18.4 \%$ \\
\hline $\mathrm{Vl}$ & 5 & 15 & $5 \%$ & $6 \%$ \\
\hline VII & 2 & 2 & $2 \%$ & $0.8 \%$ \\
\hline VIII & 9 & 9 & $9 \%$ & $3.6 \%$ \\
\hline IX & 1 & 6 & $1 \%$ & $2.4 \%$ \\
\hline$X$ & - & 6 & - & $2.4 \%$ \\
\hline XI & 2 & 1 & $2 \%$ & $0.4 \%$ \\
\hline XII & 3 & 2 & $3 \%$ & $0.8 \%$ \\
\hline XIII & - & 1 & - & $0.4 \%$ \\
\hline XIV & 3 & 15 & $3 \%$ & $6 \%$ \\
\hline$X V$ & 2 & 11 & $2 \%$ & $4.4 \%$ \\
\hline$X V I$ & 1 & 2 & $1 \%$ & $0.8 \%$ \\
\hline XVII & - & 3 & - & $1.2 \%$ \\
\hline XVIII & - & 1 & - & $0.4 \%$ \\
\hline XIX & - & 1 & - & $0.4 \%$ \\
\hline$X X$ & - & 1 & - & $0.4 \%$ \\
\hline XXI & - & 1 & - & $0.4 \%$ \\
\hline XXII & - & 2 & - & $0.8 \%$ \\
\hline XXIII & 1 & - & $1 \%$ & - \\
\hline XXIV & 1 & - & $1 \%$ & - \\
\hline$X X V$ & 2 & 1 & $2 \%$ & $0.4 \%$ \\
\hline XXVI & - & 4 & - & $1.6 \%$ \\
\hline XXVII & - & 5 & - & $2 \%$ \\
\hline XXVIII & - & 1 & - & $0.4 \%$ \\
\hline
\end{tabular}

All $p$ values in $\chi^{2}$ test or its variants (Fisher's exact test) $>0.05$; MCA - middle cerebral artery

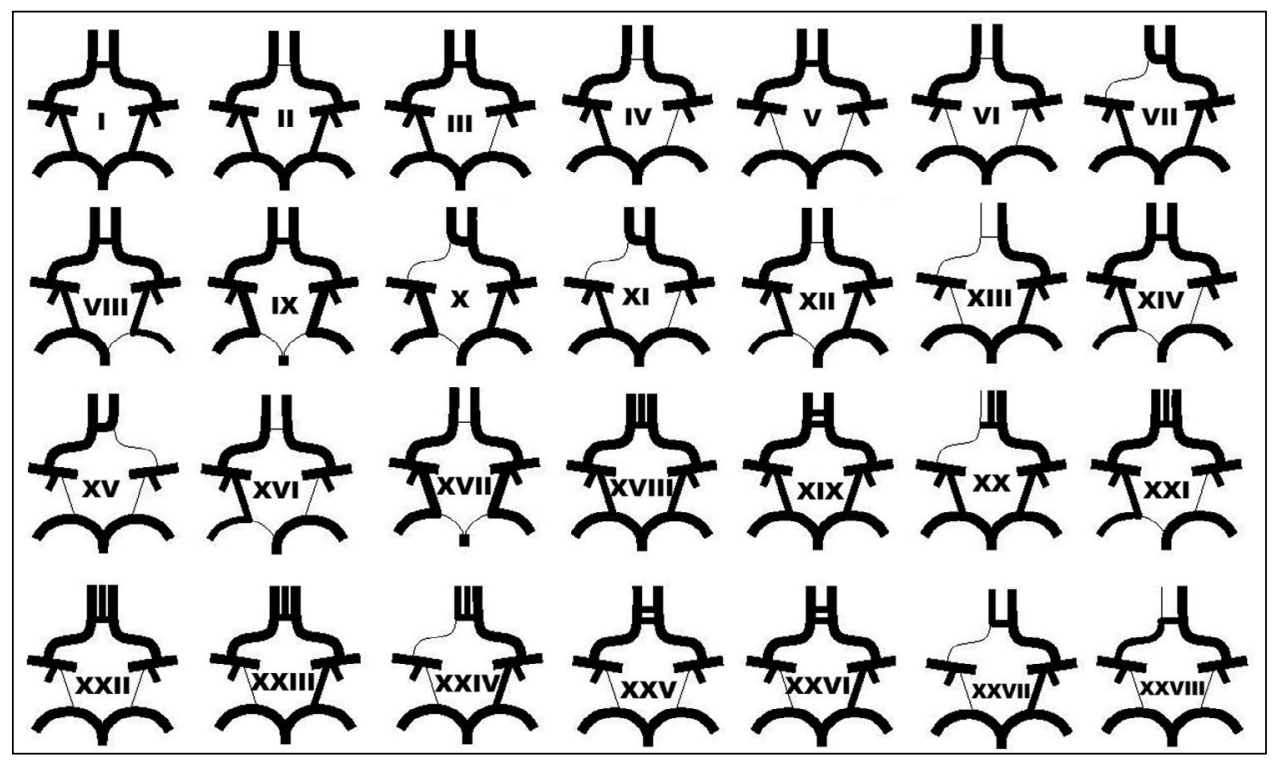

Figure 3. Circle of Willis variations. 
Table 3. Prevalence of anatomical variations of middle cerebral artery (MCA) aneurysms in patients with and without MCA aneurysms

\begin{tabular}{|c|c|c|c|}
\hline Anatomical factor & $\begin{array}{c}\text { Patients with no MCA } \\
\text { aneurysm }\end{array}$ & $\begin{array}{l}\text { Patients with MCA } \\
\text { aneurysm }\end{array}$ & $\begin{array}{c}\text { p value (Mann and } \\
\text { Whitney test) }\end{array}$ \\
\hline \multicolumn{4}{|l|}{ Left MCA } \\
\hline M1 segment length [cm] & 17 & 16.75 & $>0.05$ \\
\hline MCA length to the genu [cm] & 24.1 & 23.15 & $>0.05$ \\
\hline Distance from $\mathrm{M} 1$ division point to the genu $[\mathrm{cm}]$ & 7.1 & 7.25 & $>0.05$ \\
\hline Prevalence of bifurcation & 0.88 & 0.88 & $>0.05$ \\
\hline Prevalence of trifurcation & 0.12 & 0.12 & $>0.05$ \\
\hline Division before the MCA genu & 0.80 & 0.71 & $>0.05$ \\
\hline Division in the MCA genu & 0.18 & 0.27 & $>0.05$ \\
\hline Division after the MCA genu & 0.02 & 0.02 & $>0.05$ \\
\hline No post-division domination & 0.46 & 0.56 & $>0.05$ \\
\hline Domination of upper trunk & 0.26 & 0.12 & 0.0255 \\
\hline Domination of lower trunk & 0.24 & 0.31 & $>0.05$ \\
\hline Domination of middle trunk & 0.04 & 0.02 & $>0.05$ \\
\hline \multicolumn{4}{|l|}{ Right MCA } \\
\hline M1 segment length [cm] & 16.9 & 19.4 & $>0.05$ \\
\hline MCA length to the genu [cm] & 24.25 & 23.4 & $>0.05$ \\
\hline Distance from $\mathrm{M} 1$ division point to the genu $[\mathrm{cm}]$ & 6.65 & 5.5 & $>0.05$ \\
\hline Prevalence of bifurcation & 0.88 & 0.92 & $>0.05$ \\
\hline Prevalence of trifurcation & 0.16 & 0.08 & $>0.05$ \\
\hline Division before the MCA genu & 0.76 & 0.75 & $>0.05$ \\
\hline Division in the MCA genu & 0.21 & 0.19 & $>0.05$ \\
\hline Division after the MCA genu & 0.03 & 0.06 & $>0.05$ \\
\hline No post-division domination & 0.43 & 0.48 & $>0.05$ \\
\hline Domination of upper trunk & 0.26 & 0.17 & $>0.05$ \\
\hline Domination of lower trunk & 0.26 & 0.33 & $>0.05$ \\
\hline Domination of middle trunk & 0.04 & 0.02 & $>0.05$ \\
\hline
\end{tabular}

randomised clinical trials of mechanical thrombectomy in stroke treatment, only clots in proximal part of MCA were included. Defining the segments of MCA can be useful in deciding the eligibility of the patient for interventional treatment [4]. Currently, CTA, as noninvasive examination, is the basic tool used to assess intracranial vessels, but up to now no anatomic study of MCA was based on it. Basing the study on CTA imaging allowed creating significantly bigger database and performing more precise statistical analysis in comparison with anatomical studies based on cadavers.

In this study group the prevalence of M1 bifurcation was similar to previous studies, in which it ranged from $78 \%$ [3] to $80 \%$ [14]. In contrast to Gibo et al. [3] there were no cases of multiple division of M1 segment. It is still discussed, what should be recognised as normal MCA anatomy. Most of the authors consider both bifurcation and trifurcation as normal anatomy. However, some authors see "pseudotrifurcations" as an early origin of medial trunk directly after bifurcation [7].

Distance from terminal ICA division to the first division of MCA was similar to the results reported by Umansky et al. [21] - $15.1 \mathrm{~mm}$ and slightly shorter than in a more recent study by Pai et al. [14] - $20 \mathrm{~mm}$.

Prevalence of MCA anomalies was similar to former studies. In microsurgical studies the prevalence ranged from $1 \%$ to $3 \%[1,3,6]$ for MCA duplication. In case of accessory MCA the prevalence ranged from $2 \%$ to $2.7 \%[19,21]$ - slightly more than in this study. Fenestration was an extremely rare occurrence, with maximum prevalence of $1 \%$ [21]. In this study group there was no case of MCA with one M2 trunk, which Umansky et al. [21] observed in 4\% of the cases, but was not mentioned in any other study. 
Mirror aneurysms were found in $10 \%$ of patients, which is similar to earlier results [12].

The aim of this study was also to examine whether anatomical variations of MCA and the circle of Willis have impact on formation of MCA aneurysms. It has been proved on theoretical models that changes in the circle of Willis lead to increased shear stress in MCA [13]. It is also known, that shear stress is one of the most important factors influencing aneurysm formation. Underdevelopment of the circle of Willis, which leads to its anatomical variations, can also contribute to weakening of its arteries [18]. It remains significant field of study, with newer research highlighting influence of circle of Willis variations on results of aneurysms treatment [20]. Our results of circle of Willis variations are similar to earlier studies [10]. However, this study failed to demonstrate statistically significant correlation of anatomical variations of the circle of Willis and prevalence of MCA aneurysms. Assessment of the circle of Willis cannot be used to identify patients with increased risk of MCA aneurysm formation based on CTA of head.

The study also failed to show correlation between anatomical variations of MCA and increased prevalence of MCA aneurysms. Higher prevalence of upper trunk domination in patients with aneurysms can be caused by small group of patients with this variation. Other authors have only found correlation on increased aneurysm prevalence with small angle between M2 trunks after M1 division.

\section{CONCLUSIONS}

The most common configuration of MCA is bifurcation before the genu with no dominating post-division trunk. There are no differences in MCA anatomy between both hemispheres and no correlation between variations of Circle of Willis and MCA. Incidence of MCA aneurysms is not correlated with anatomical variations of MCA and the circle of Willis.

\section{REFERENCES}

1. Crompton MR. The pathology of ruptured middle-cerebral aneurysms with special reference to the differences between the sexes. Lancet. 1962; 2(7253): 421-425, indexed in Pubmed: 13882322 .

2. Elsharkawy $A$, Lehečka $M$, Niemelä $M$, et al. A new, more accurate classification of middle cerebral artery aneurysms: computed tomography angiographic study of 1,009 consecutive cases with 1,309 middle cerebral artery aneurysms. Neurosurgery. 2013; 73(1): 94-102; discussion 102. doi: $10.1227 / 01$ neu.0000429842.61213.d5, indexed in Pubmed: 23615110.

3. Gibo H, Carver CC, Rhoton AL, et al. Microsurgical anatomy of the middle cerebral artery. J Neurosurg. 1981; 54(2): 151-169, doi: 10.3171/jns.1981.54.2.0151, indexed in Pubmed: 7452329.
4. Goyal M, Menon BK, Krings $\mathrm{T}$, et al. What constitutes the M1 segment of the middle cerebral artery? J Neurointerv Surg. 2016 [Epub ahead of print], doi: 10.1136/neurintsurg-2015-012191, indexed in Pubmed: 26863104.

5. Huttunen T, von und zu Fraunberg M, Frösen J, et al. Saccular intracranial aneurysm disease: distribution of site, size, and age suggests different etiologies for aneurysm formation and rupture in 316 familial and 1454 sporadic eastern Finnish patients. Neurosurgery. 2010; 66(4): 631-8; discussion 638, doi: 10.1227/01.NEU.0000367634.89384.4B, indexed in Pubmed: 20190670.

6. Jain KK. Some observations on the anatomy of the middle cerebral artery. Can J Surg. 1964; 7: 134-139, indexed in Pubmed: 14142582

7. Kahilogullari G, Ugur HC, Comert A, et al. The branching pattern of the middle cerebral artery: is the intermediate trunk real or not? An anatomical study correlating with simple angiography. J Neurosurg. 2012; 116(5): 1024-1034, doi: $10.3171 / 2012.1 . J N S 111013$, indexed in Pubmed: 22360571

8. Kang HG, Kim BJ, Lee J, et al. Risk factors associated with the presence of unruptured intracranial aneurysms. Stroke. 2015; 46(11): 3093-3098, doi: 10.1161/STROKEAHA.115.011351, indexed in Pubmed: 26451026.

9. Kathuria S, Gregg L, Chen J, et al. Normal cerebral arterial development and variations. Semin Ultrasound CT MR. 2011; 32(3): 242-251, doi: 10.1053/j.sult.2011.02.002, indexed in Pubmed: 21596279

10. Klimek-Piotrowska W, Kopeć M, Kochana M, et al. Configurations of the circle of Willis: a computed tomography angiography based study on a Polish population. Folia Morphol. 2013; 72(4): 293-299, indexed in Pubmed: 24402749.

11. Krasny A, Nensa F, Sandalcioglu IE, et al. Association of aneurysms and variation of the $A 1$ segment. J Neurointerv Surg. 2014; 6(3): 178-183, doi: 10.1136/neurintsurg-2013-010669, indexed in Pubmed: 23612892.

12. Meissner I, Torner J, Huston J, et al. International Study of Unruptured Intracranial Aneurysms Investigators. Mirror aneurysms: a reflection on natural history. J Neurosurg. 2012; 116(6): 1238-1241, doi: 10.3171/2012.1.JNS11779, indexed in Pubmed: 22404675.

13. Nam SW, Choi S, Cheong Y, et al. Evaluation of aneurysmassociated wall shear stress related to morphological variations of circle of Willis using a microfluidic device. J Biomech. 2015; 48(2): 348-353, doi: 10.1016/j.jbiomech.2014.11.018, indexed in Pubmed: 25497378.

14. Pai SB, Varma RG, Kulkarni RN. Microsurgical anatomy of the middle cerebral artery. Neurol India. 2005; 53(2): 186-190, indexed in Pubmed: 16010057.

15. Papazova M, Zhivadinovik J, Trpkovska B, et al. Anatomy of middle cerebral artery. . Acta Morphol. 2012; 9(1): 5-9.

16. Słowik A, Wnuk M, Brzegowy P, et al. Polish Thrombectomy Initiative. Mechanical thrombectomy in acute stroke - Five years of experience in Poland. Neurol Neurochir Pol. 2017; 51(5): 339-346, doi: 10.1016/j.pjnns.2017.05.004, indexed in Pubmed: 28756015.

17. Rinne J, Hernesniemi J, Niskanen M, et al. Analysis of 561 patients with 690 middle cerebral artery aneurysms: anatomic and clinical features as correlated to management outcome. Neurosurgery. 1996; 38(1): 2-11, indexed in Pubmed: 8747945.

18. Songsaeng D, Geibprasert S, Willinsky $R$, et al. Impact of anatomical variations of the circle of Willis on the incidence of aneurysms and their recurrence rate following endovascular treatment. Clin Radiol. 2010; 65(11): 895-901, doi: 10.1016/j. crad.2010.06.010, indexed in Pubmed: 20933644.

19. Tacconi L, Johnston FG, Symon L. Accessory middle cerebral artery. Case report. J Neurosurg. 1995; 83(5): 916-918, doi: 10.3171/jns.1995.83.5.0916, indexed in Pubmed: 7472565.

20. Tarulli E, Sneade M, Clarke A, et al. Effects of circle of Willis anatomic variations on angiographic and clinical outcomes of coiled anterior communicating artery aneurysms. AJNR Am J Neuroradiol. 2014; 35(8): 1551-1555, doi: 10.3174/ ajnr.A3991, indexed in Pubmed: 24948501.

21. Umansky F, Dujovny M, Ausman Jl, et al. Anomalies and variations of the middle cerebral artery: a microanatomica study. Neurosurgery. 1988; 22(6 Pt 1): 1023-1027, indexed in Pubmed: 3047592. 